\title{
Internal representations of smell in the Drosophila brain
}

\author{
Hui-Hao Lin ${ }^{1}$, Chih-Yung Lin ${ }^{1} \&$ Ann-Shyn Chiang ${ }^{1,2,3, *}$ \\ ${ }^{1}$ Institute of Biotechnology and Department of Life Science, National Tsing Hua University, Hsinchu, 30013, \\ Taiwan, R.O.C.; ${ }^{2}$ Brain Research Center, National Tsing Hua University, University System of Taiwan, \\ Hsinchu, 30013, Taiwan, R.O.C.; ${ }^{3}$ Cold Spring Harbor Laboratory, 1 Bungtown Road, Cold Spring Harbor, \\ NY, 11724, USA
}

Received 9 March 2007; accepted 14 March 2007

(c) 2007 National Science Council, Taipei

Key words: Drosophila, Brain circuit, Olfaction, Mushroom body, Map

\begin{abstract}
Recent advances in sensory neuroscience using Drosophila olfaction as a model system have revealed brain maps representing the external world. Once we understand how the brain's built-in capability generates the internal olfactory maps, we can then elaborate how the brain computes and makes decision to elicit complex behaviors. Here, we review current progress in mapping Drosophila olfactory circuits and discuss their relationships with innate olfactory behaviors.
\end{abstract}

\section{Introduction}

All animals are born with a priori capabilities that are hardwired into the nervous system for instinctive behaviors independent of experience. The internal representation of the external world is then evolved as a result of the brain's built-in capability to derive meaning from information in the surrounding environment. A cognitive map is therefore established through the experiencedependent modifications in the prewired brain circuits. To elicit adaptive behavioral responses, the spatiotemporal pattern of sensory stimuli perceived by an animal must be ultimately translated into neural codes in the brain. How information of the outside world is transmitted to the inside of the brain? This big question has been extensively addressed in a small but functional neural circuit: the olfactory system of the fruit fly, Drosophila melanogaster. Drosophila exhibits

*To whom correspondence should be addressed. Fax: +1-3571-7237; E-mail: aschiang@life.nthu.edu.tw

Hui-Hao lin and Chih-Yung Lin contributed equally to this work. many complex behaviors controlled by an olfactory system that has been proven to be genetically and anatomically similar to those in the vertebrates [1-5]. Amenability of the genetic toolbox in the Drosophila allows manipulation of identified brain neurons with such a spatial and temporal precision that results in unprecedented insight into the design and function of the brain. Advances in Drosophila sensory neuroscience are clarifying information processing mechanisms that underlie a response in the brain to a stimulus [6]. Here, we limit our discussion on the current progress in mapping brain circuits involved in the Drosophila olfactory behaviors. Understanding the neural maps representing olfaction will have a great impact on the insight of molecular and cellular mechanisms involving in the olfactory memory formation $[7,8]$.

\section{Organization of the Drosophila olfactory circuitry}

Figure 1 shows the organization of the Drosophila olfactory circuitry. Odors are detected by a large family of odorant receptors (ORs) expressed in 


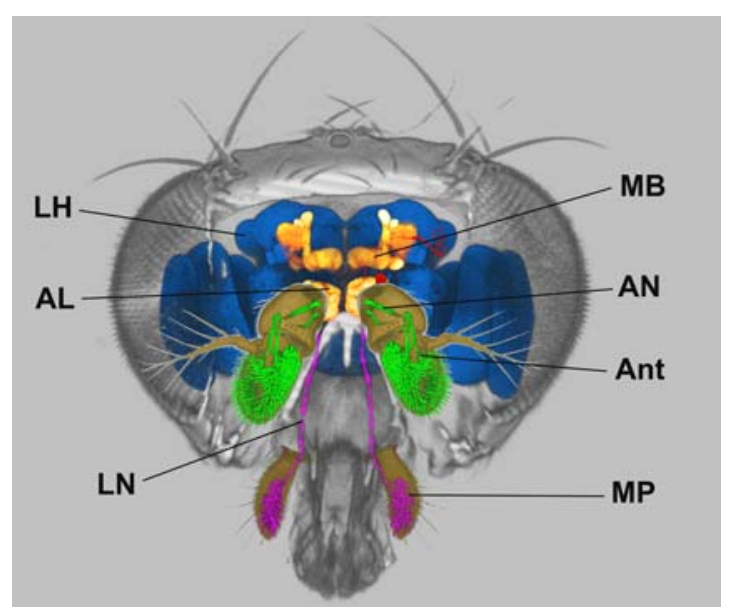

Figure 1. Organization of the Drosophila olfactory system. Odorants are detected by the olfactory sensory neurons (OSNs) located on the antenna (Ant, green) and maxillary palp (MP, magenta) sending their axons via the antennal nerve $(\mathrm{AN})$ and the labial nerve $(\mathrm{LN})$, respectively, to the antennal lobe (AL, orange); where the projection neurons (PNs, red) receive the information and relay to the mushroom body (MB) and the lateral horn (LH). This picture is a montage of four sets of confocal images taking from the whole head (grey), brain (blue), antenna (Ant), and maxillary palp (MP) separately from different flies. The head image is derived from auto-fluorescence excited with $633 \mathrm{~nm}$ laser. MB and AL (orange) are segmented from the whole brain stained by nc82 antibody. OSNs in the antenna and MPs are labeled by OR83b-GFP. The single PN image is derived from MARCM visualization of GH146-GAL4.

olfactory sensory neurons (OSNs) on the maxillary palp and the antenna [9]. Individual OSNs express only one, or a few, but rarely, OR on the ciliated endings of OSN dendrites [10-12]. The axons of OSNs expressing the same OR converge to a single glomerulus in the ipsilateral antennal lobe (AL) [13-15], where they synapse with projection neurons (PNs) that project to the mushroom body (MB), the lateral horn ( $\mathrm{LH})[9]$ and inhibitory and excitatory local neurons (LNs), that might modulate the information among glomeruli $[9,10,16]$.

\section{The OR-to-OSN map}

Drosophila has a family of $60 \mathrm{OR}$ genes that encode 62 odorant receptors determining the odorresponse profile of the OSNs [14, 17-20]. Each OR gene expresses in a subset of OSNs except Or83b that acts as a co-receptor in ca. $70 \%$ OSNs $[20$ 23]. Flies lacking OR 83 b display severe defects in many different olfactory behaviors but with their lifespan extended [22, 24]. In Drosophila larva, each of the paired olfactory dorsal organs houses 21 OSNs. Each OSN expresses a single OR in addition to OR83b, including 11 ORs that are not expressed in the adult [25-27]. In Drosophila adult, the recognition of odors is accomplished by sensory hairs distributed over the surface of the third antennal segment and the maxillary palp. There are about 2600 OSNs housed inside three different types of hair-like sensilla: the club-shaped basiconic, the long-pointed trichoid and the short coeloconic sensilla [28-31]. The OSNs housed inside the distal-lateral trichoid and the proximomedial basiconic sensilla project their axons to lateral-anterior and proximo-medial AL glomerul$\mathrm{i}$, respectively [32-34]. In the maxillary palp, OSNs projecting via the labial nerve to the AL are all contained in the basiconic sensilla. Every individual flies has about 1000 sensilla, each contains 1-4 OSNs [35]. The bipolar OSNs project dendrites in sensillar lymph interacting with odorants and project axons to the AL, where olfactory information is processed. In the sensillar lymph, olfactory binding proteins secreted by the support cells may act as transport vehicles for the odorants to bind with the OR. Odorant-OR binding then activates a heterotrimeric G-protein that influence the action potentials transmitted via axons to the AL [36-38]. Intriguingly, a single ORN can exhibit both stimulatory and inhibitory responses to different odorants [39]. A chemotopic map of OR responses to 110 odorants indicates that OSN responses are chemical class-dependent [39, 40].

\section{The OSN-to-PN map}

In the ALs, the axons of OSNs project to about 50 glomeruli, where they synapse with dendrites of intrinsic LNs and output PNs. Axons of OSNs with the same OR expression converge to the same AL glomerulus suggesting stereotypic connectivity with PNs [15, 41-43]. Co-convergence to VA6 glomerulus from two different classes of OSNs labeled by different OR Gal4 lines is an exception $[32,33]$. Therefore, a topographic map of OR responses in the peripheral sensory organs is further represented in the ALs [44]. Stereotypic connectivity between the OSNs and PNs has been confirmed by functional calcium imaging [20] and 2-deoxy glucose mapping $[45,46]$ showing that 
different odorants elicit defined glomerular activities. In the ALs, odor responses of the PNs are modulated by local neurons that form widespread intra- as well as inter-glomerular connections among many glomeruli [16, 47-50]. It has been proposed that odor coding is sharpened at ALs by GABAergic LNs that inhibit background signals from adjacent glomeruli [49]. However, this can not explain why PNs are more broadly tuned to different odors than OSNs [20]. A newly discovered population of cholinergic LNs with multiglomerular processes responds broadly to odors [16] suggesting that PNs receive information from a combination of glomerulus-specific OSN afferents and "diffused" LN excitation [16].

\section{The PN-to-LH map}

PNs convey olfactory information from paired ALs to high brain centers, $\mathrm{MB}$ and $\mathrm{LH}$, via three different tracks: the inner antenna-cerebrum track (iACT), the medial ACT (mACT), the outer ACT (oACT). One AL has about 50 glomeruli, each innervated by dendrites of 3-7 PNs [44, 51, 52]. As a general principle, one $\mathrm{PN}$ sends dendrites into only a single glomerulus. PN axons may directly project to $\mathrm{LH}$ via mACT and oACT. Most PNs, however, project in parallel via iACT to the $\mathrm{LH}$ en route MB calyx. A typical PN usually gives 1-4 branches in the calyx and has many termini in the LH. How do the PNs carry olfactory information to the high brain centers? Using genetic mosaic FLP-out technique, visualization of individual PNs has revealed that the axonal branch patterns of PNs coming from the same AL glomerulus display stereotypy in the $\mathrm{LH}$ among different flies $[51,52]$. These axonal termini of PNs in the $\mathrm{LH}$ appear to segregate into three distinct spatial domains: anterior ventral, posterior dorsal and lateral ventral regions [53]. Visualization of gene expression patterns from more than 4000 GAL4 lines have revealed three different classes of $\mathrm{LH}$ local neurons that have projection patterns corresponding to the distribution of $\mathrm{PN}$ axonal termini [53]. These results suggest a stereotypic connectivity between specific PNs and specific LH neurons via iACT and mACT. If so, a topographic map of olfactory responses in the ALs is further represented in the LH [53]. It has not been addressed if PNs via oACT also give stereotyped termini in the
LH and if their types are different from those via iACT and mACT.

\section{The PN-to-KC map}

The spatial distribution of PN axon branching patterns is highly stereotyped in the LH but less clear in the MB [51, 52]. The paired MBs play an essential role in insect olfactory behaviors [54]. One MB in adult Drosophila consists of $\sim 2500$ intrinsic Kenyon cells (KCs) derived from four neuroblasts $[55,56]$, each of which sequentially generates five distinct classes of KCs: $\gamma, \alpha^{\prime} \beta^{\prime}$, pioneer $\alpha \beta$, early $\alpha \beta$ and late $\alpha \beta$ respectively [57, 58]. KCs give dendrites exclusively in the calyx, where they synapse with PN axonal termini. KC axons constitute a massive parallel tract called peduncle that extends ventral-frontally and splits at its distal end: one projecting dorsally and the other medially. The dorsal projection is composed of $\alpha$ and $\alpha^{\prime}$ lobes, and the medial projection is composed of $\beta, \beta^{\prime}$ and $\gamma$ lobes [59]. Stereotyped odor-evoked activity occurs both in the $\mathrm{AL}$ glomeruli $[20,60]$ and the MB calyx [61]. As odor concentration increase, more glomeruli are activated in the AL and more KCs are activated in the calyx [20,61]. PN axonal termini appear to form three concentric zones in the MB calyx [53]. Analysis of topographic connectivity between PNs and KCs in the MB calyx has been hampered due to insufficient optical resolution and lack of anatomical landmarks. The dual MARCM technique allowing visualization of individual PNs and KCs in two different colors may help to answer this question [62].

\section{Olfactory information processing for innate behaviors}

Innate behaviors, such as courtship ritual, are instinctive actions of an animal without prior experience. These inherited programs offer a unique opportunity to use genetic and anatomic analysis to dissect and characterize the neural substrates of complex behaviors. In insects, avoidance or attraction to different odors is essential for the exploitation of food sources, selection of mates and escaping from harmful situations. A central question in olfaction is how the brain discriminates 
different odors to elicit an appropriate behavioral response. In Drosophila, stereotypic connectivity maps of OR-to-OSN [39], OSN-to-PN [32, 33], PN-to-LH [51, 52] and PN-to-KC [61] begin to reveal a hierarchy of information processing with increasing convergence, which enable higher-order centers to integrate information relayed separately from lower-order neurons. Although stereotypic connectivity maps from OSNs to PNs to KCs give the impression of a straight and simple path, neural computation for odor discrimination clearly requires multiple steps of information integration as it makes its way through the brain. A single OSN can exhibit both excitatory and inhibitory responses to different odorants [40]. In the ALs, odor responses of the PNs are modulated by inhibitory as well as stimulatory local neurons $[16,50]$. In the MBs, KCs may receive both stimulatory and inhibitory stimuli from PNs since some of them are cholinergic but others are GABAergic [49]. Immunohistochemical labeling and GFP expression patterns in Cha-GAL4 and GAD-GAL4 lines indicate that $\mathrm{KCs}$ are also composed of both cholinergic and GABAergic neurons (Lin and Chiang, unpublished observation). Such complexity of odor representations and information integration hierarchies greatly reduces the possibility of overlap between spatiotemporal patterns elicited by two different odorants, making them easier to be discriminated or memorized and recalled [63].

A Drosophila uses $\mathrm{CO}_{2}$ released by other stressed flies as an alarm signal [64]. $\mathrm{CO}_{2}$ is sensed by antennal OSNs via a pair of chemosensory receptors, Gr21a and Gr63a [65]. $\mathrm{CO}_{2}$-responsive neurons relay information to only a single glomerulus in the AL, the V glomerulus [64]. Inhibition of synaptic transmission in the $\mathrm{CO}_{2}$-responsive neurons, using a temperaturesensitive shibire gene, blocks the avoidance response to $\mathrm{CO}_{2}$. It remains unclear how the brain sense and apply the $\mathrm{CO}_{2}$-induced signals in computation. Chemical ablation of the MBs abolishes olfactory attraction but not repulsion behaviors suggesting that the computation center for the attractive odors is likely at the MB and for the repulsive odors is likely at the LH [66]. Consistently, blocking neurotransmitter release from MBs using the temperature sensitive shibire has no effect on $\mathrm{CO}_{2}$ avoidance behavior [64], implying that $\mathrm{LH}$ may involve in computing the $\mathrm{CO}_{2}$-induced signals. However, this hypothesis remains to be verified since PNs connecting $\mathrm{V}$ glomerulus to higher brain centers have not been mapped and LH specific drivers are still unavailable for the manipulation of its biological functions.

Drosophila courtship represents the most complex innate behavior. A courting male performs a stereotypical sequence of ritual activities beginning with attracting to a receptive female and then he orients, taps, sings, licks and finally bends his abdomen to copulation [67]. Males exhibit homosexual courtship if a transcription factor gene called fruitless (fru) has defect. Although the roles of fru remain unclear, neurotransmission from brain neurons expressing fru is essential for normal courtship behavior [68]. Intriguingly, fru-expressing neurons appear to interconnect with each other, as indicated by that fru-expressing OSNs and PNs innervate the same set of AL glomeruli (VL2a, DA1, VA1 and VA6). fru expresses also in the MBs of the $\gamma$ and $\alpha / \beta$ lobes, and courtship conditioning is impaired when the expression of male-specific fru transcript is disrupted in MB $\gamma$ neurons [69]. A recent study indicates that fru is also associated with patterns of aggressive behavior that is sex-specific and with the formation of dominance rank in a group of flies [70]. These finding suggest that, aggression and mating are closely intertwined in Drosophila, as also in mammals [71, 72].

\section{Concluding remarks}

How the olfactory circuitry executes various different programs of innate behaviors? Olfactory information converges from OSNs to PNs in the $\mathrm{AL}$ and then diverges from PNs to KCs of the MB and principle neurons of the LH suggesting that information processing must perform in the AL to translate peripheral odor stimuli into a neural coding intelligible to neurons of high brain centers. Where the MB and $\mathrm{LH}$ send the processed information to execute specific behavior outputs? Are there other higher brain centers involving olfactory information processing? Screening mutants with defects in olfactory behaviors have identified a set of genes involving in particular olfactory innate behaviors [65, 68]. Mapping the expression of these olfactory behavior essential genes should reveal additional, if any, brain 
circuits involving in the computation of olfactory information. Each step of information processing in the hierarchy can then be characterized by the manipulation of neuronal activities and gene expressions with various genetic tools available. Identification of genes (intracellular hierarchy) and circuits (intercellular hierarchy) involving in particular olfactory behaviors promises to reveal the secret of the brain's operations in the information acquisition, processing, transmission, storage, and retrieval.

\section{Acknowledgements}

We thank Drs. Chang-Huain Hsieh and HsiuMing Chang for critical reading and discussion. This work was supported by grants from $\mathrm{Na}$ tional Science Council, Brain Research Center of the University System of Taiwan, and the National Center for High-performance Computing.

\section{References}

1. Hildebrand J.G. and Shepherd G.M., Mechanisms of olfactory discrimination: converging evidence for common principles across phyla. Annu. Rev. Neurosci. 20: 595-631, 1997.

2. Laurent G., Stopfer M., Friedrich R.W., Rabinovich M.I., Volkovskii A. and Abarbanel H.D., Odor encoding as an active, dynamical process: experiments, computation, and theory. Annu. Rev. Neurosci. 24: 263-297, 2001.

3. Lessing D. and Carlson J.R., Chemosensory behavior: the path from stimulus to response. Curr. Opin. Neurobiol. 9(6) 766-771, 1999.

4. Mombaerts P., How smell develops. Nat. Neurosci. 4(Suppl) 1192-1198, 2001.

5. Vosshall L.B., Olfaction in Drosophila. Curr. Opin. Neurobiol. 10(4) 498-503, 2000.

6. Bargmann C.I., Comparative chemosensation from receptors to ecology. Nature 444(7117) 295-301, 2006.

7. Davis R.L., Olfactory memory formation in Drosophila: from molecular to systems neuroscience. Annu. Rev. Neurosci. 28: 275-302, 2005.

8. Margulies C., Tully T. and Dubnau J., Deconstructing memory in Drosophila. Curr. Biol. 15(17) 700-713, 2005.

9. Mombaerts P., Seven-transmembrane proteins as odorant and chemosensory receptors. Science 286(5440) 707-711, 1999.

10. Barnea G., O'Donnell S., Mancia F., Sun X., Nemes A., Mendelsohn M. and Axel R., Odorant receptors on axon termini in the brain. Science 304(5676) 1468, 2004.

11. Dobritsa A.A., van der van Goes Naters W., Warr C.G., Steinbrecht R.A. and Carlson J.R., Integrating the molecular and cellular basis of odor coding in the Drosophila antenna. Neuron 37(5) 827-841, 2003.
12. Elmore T. and Smith D.P., Putative Drosophila odor receptor OR43b localizes to dendrites of olfactory neurons. Insect. Biochem. Mol. Biol. 31(8) 791-798, 2001.

13. Belluscio L., Gold G.H., Nemes A. and Axel R., Mice deficient in G(olf) are anosmic. Neuron 20(1) 69-81, 1998.

14. Bozza T., Feinstein P., Zheng C. and Mombaerts P., Odorant receptor expression defines functional units in the mouse olfactory system. J. Neurosci. 22(8) 3033-3043, 2002.

15. Vosshall L.B., Amrein H., Morozov P.S., Rzhetsky A. and Axel R., A spatial map of olfactory receptor expression in the Drosophila antenna. Cell 96(5) 725-736, 1999.

16. Shang Y., Claridge-Chang A., Sjulson L., Pypaert M. and Miesenbock G., Excitatory local circuits and their implications for olfactory processing in the fly antennal lobe. Cell 128(3) 601-612, 2007.

17. Hallem E.A., Ho M.G. and Carlson J.R., The molecular basis of odor coding in the Drosophila antenna. Cell 117(7) 965-979, 2004.

18. Malnic B., Hirono J., Sato T. and Buck L.B., Combinatorial receptor codes for odors. Cell 96(5) 713-723, 1999.

19. Oka Y., Omura M., Kataoka H. and Touhara K., Olfactory receptor antagonism between odorants. EMBO. J. 23(1) 120-126, 2004.

20. Wang J.W., Wong A.M., Flores J., Vosshall L.B. and Axel R., Two-photon calcium imaging reveals an odor-evoked map of activity in the fly brain. Cell. 112(2) 271-282, 2003.

21. Benton R., Sachse S., Michnick S.W. and Vosshall L.B., Atypical membrane topology and heteromeric function of Drosophila odorant receptors in vivo. PloS. Biol. 4(2) e20, 2006.

22. Larsson M.C., Domingos A.I., Jones W.D., Chiappe M.E., Amrein H. and Vosshall L.B., Or83b encodes a broadly expressed odorant receptor essential for Drosophila olfaction. Neuron 43(5) 703-714, 2004.

23. Ng M., Roorda R.D., Lima S.Q., Zemelman B.V., Morcillo P. and Miesenbock G., Transmission of olfactory information between three populations of neurons in the antennal lobe of the fly. Neuron 36(3) 463-474, 2002.

24. Neuhaus E.M., Gisselmann G., Zhang W., Dooley R., Stortkuhl K. and Hatt H., Odorant receptor heterodimerization in the olfactory system of Drosophila melanogaster. Nat. Neurosci. 8(1) 15-17, 2005.

25. Kreher S.A., Kwon J.Y. and Carlson J.R., The molecular basis of odor coding in the Drosophila larva. Neuron 46(3) 445-456, 2005.

26. Python F. and Stocker R.F., Adult-like complexity of the larval antennal lobe of $D$. melanogaster despite markedly low numbers of odorant receptor neurons. J. Comp. Neurol. 445(4) 374-387, 2002.

27. Ramaekers A., Magnenat E., Marin E.C., Gendre N., Jefferis G.S., Luo L. and Stocker R.F., Glomerular maps without cellular redundancy at successive levels of the Drosophila larval olfactory circuit. Curr. Biol. 15(11) 982 992, 2005.

28. Goulding S.E., zur Lage P. and Jarman A.P., Amos, a proneural gene for Drosophila olfactory sense organs that is regulated by lozenge. Neuron 25(1) 69-78, 2000.

29. Jhaveri D., Sen A., Reddy G.V. and Rodrigues V., Sense organ identity in the Drosophila antenna is specified by the expression of the proneural gene atonal. Mech. Dev. 99(12) 101-111, 2000.

30. Stocker R.F. and Gendre N., Peripheral and central nervous effects of lozenge3: a Drosophila mutant lacking basiconic antennal sensilla. Dev. Biol. 127(1) 12-24, 1988. 
31. zur Lage P.I., Prentice D.R., Holohan E.E. and Jarman A.P., The Drosophila proneural gene amos promotes olfactory sensillum formation and suppresses bristle formation. Development 130(19) 4683-4693, 2003.

32. Couto A., Alenius M. and Dickson B.J., Molecular, anatomical, and functional organization of the Drosophila olfactory system. Curr. Biol. 15(17) 1535-1547, 2005.

33. Fishilevich E. and Vosshall L.B., Genetic and functional subdivision of the Drosophila antennal lobe. Curr. Biol. 15(17) 1548-1553, 2005.

34. Jefferis G.S., Insect olfaction: a map of smell in the brain. Curr. Biol. 15(17) 668-670, 2005.

35. Stocker R.F., Drosophila as a focus in olfactory research: mapping of olfactory sensilla by fine structure, odor specificity, odorant receptor expression, and central connectivity. Microsc. Res. Tech. 55(5) 284-296, 2001.

36. Firestein S., How the olfactory system makes sense of scents. Nature 413(6852) 211-218, 2001.

37. McCudden C.R., Hains M.D., Kimple R.J., Siderovski D.P. and Willard F.S., G-protein signaling: back to the future. Cell. Mol. Life. Sci. 62(5) 551-577, 2005.

38. Xu P., Atkinson R., Jones D.N. and Smith D.P., Drosophila OBP LUSH is required for activity of pheromonesensitive neurons. Neuron 45(2) 193-200, 2005.

39. Hallem E.A. and Carlson J.R., Coding of odors by a receptor repertoire. Cell 125(1) 143-160, 2006.

40. Hallem E.A. and Carlson J.R., The odor coding system of Drosophila. Trends. Genet. 20(9) 453-459, 2004.

41. Clyne P.J., Warr C.G., Freeman M.R., Lessing D., Kim J. and Carlson J.R., A novel family of divergent seventransmembrane proteins: candidate odorant receptors in Drosophila. Neuron 22(2) 327-338, 1999.

42. Gao Q. and Chess A., Identification of candidate Drosophila olfactory receptors from genomic DNA sequence. Genomics 60(1) 31-39, 1999.

43. Robertson H.M., Warr C.G. and Carlson J.R., Molecular evolution of the insect chemoreceptor gene superfamily in Drosophila melanogaster. Proc. Natl. Acad. Sci. USA 100(2) 14537-14542, 2003.

44. Jefferis G.S., Marin E.C., Stocker R.F. and Luo L., Target neuron prespecification in the olfactory map of Drosophila. Nature 414(6860) 204-208, 2001.

45. Rodrigues V., Spatial coding of olfactory information in the antennal lobe of Drosophila melanogaster. Brain. Res. 453(1-2) 299-307, 1988.

46. Rodrigues V. and Buchner E., [3H]2-deoxyglucose mapping of odor-induced neuronal activity in the antennal lobes of Drosophila melanogaster. Brain. Res. 324(2) 374378, 1984.

47. Laissue P.P., Reiter C., Hiesinger P.R., Halter S., Fischbach K.F. and Stocker R.F., Three-dimensional reconstruction of the antennal lobe in Drosophila melanogaster. J. Comp. Neurol. 405(4) 543-552, 1999.

48. Stocker R.F., The organization of the chemosensory system in Drosophila melanogaster: a review. Cell. Tissue. Res. 275(1) 3-26, 1994.

49. Wilson R.I. and Laurent G., Role of GABAergic inhibition in shaping odor-evoked spatiotemporal patterns in the Drosophila antennal lobe. J. Neurosci. 25(40) 9069-9079, 2005.

50. Wilson R.I., Turner G.C. and Laurent G., Transformation of olfactory representations in the Drosophila antennal lobe. Science 303(5656) 366-370, 2004.
51. Marin E.C., Jefferis G.S., Komiyama T., Zhu H. and Luo L., Representation of the glomerular olfactory map in the Drosophila brain. Cell 109(2) 243-255, 2002.

52. Wong A.M., Wang J.W. and Axel R., Spatial representation of the glomerular map in the Drosophila protocerebrum. Cell 109(2) 229-241, 2002.

53. Tanaka N.K., Awasaki T., Shimada T. and Ito K., Integration of chemosensory pathways in the Drosophila secondorder olfactory centers. Curr. Biol. 14(6) 449-457, 2004.

54. Heisenberg M., Mushroom body memoir: from maps to models. Nat. Rev. Neurosci. 4(4) 266-275, 2003.

55. Technau G. and Heisenberg M., Neural reorganization during metamorphosis of the corpora pedunculata in Drosophila melanogaster. Nature 295(5848) 405-407, 1982.

56. Ito K., Awano W., Suzuki K., Hiromi Y. and Yamamoto D., The Drosophila mushroom body is a quadruple structure of clonal units each of which contains a virtually identical set of neurons and glial cells. Development 124(4) 761-771, 1997.

57. Lee T., Lee A. and Luo L., Development of the Drosophila mushroom bodies: sequential generation of three distinct types of neurons from a neuroblast. Development 126(18) 4065-4076, 1999.

58. Zhu S., Chiang A.S. and Lee T., Development of the Drosophila mushroom bodies: elaboration, remodeling and spatial organization of dendrites in the calyx. Development 130(12) 2603-2610, 2003.

59. Crittenden J.R., Skoulakis E.M., Han KA., Kalderon D. and Davis R.L., Tripartite mushroom body architecture revealed by antigenic markers. Learn. Mem. 5(1-2) 38-51, 1998.

60. Yu D., Ponomarev A. and Davis R.L., Altered representation of the spatial code for odors after olfactory classical conditioning; memory trace formation by synaptic recruitment. Neuron 42(3) 437-449, 2004.

61. Wang Y., Guo H.F., Pologruto T.A., Hannan F., Hakker I., Svoboda K. and Zhong Y., Stereotyped odor-evoked activity in the mushroom body of Drosophila revealed by green fluorescent protein-based $\mathrm{Ca} 2+$ imaging. J. Neurosci. 24(29) 6507-6514, 2004.

62. Lai S.L. and Lee T., Genetic mosaic with dual binary transcriptional systems in Drosophila. Nat. Neurosci. 9(5) 703-709, 2006.

63. Stopfer M., Olfactory coding: inhibition reshapes odor responses. Curr. Biol. 15(24) 996-998, 2005.

64. Suh G.S., Wong A.M., Hergarden A.C., Wang J.W., Simon A.F., Benzer S., Axel R. and Anderson D.J., A single population of olfactory sensory neurons mediates an innate avoidance behaviour in Drosophila. Nature 431(7010) 854 $859,2004$.

65. Jones W.D., Cayirlioglu P., Kadow I.G. and Vosshall L.B., Two chemosensory receptors together mediate carbon dioxide detection in Drosophila. Nature 445(7123) 86-90, 2007.

66. Wang Y., Chiang A.S., Xia S., Kitamoto T., Tully T. and Zhong Y., Blockade of neurotransmission in Drosophila mushroom bodies impairs odor attraction, but not repulsion. Curr. Biol. 13(21) 1900-1904, 2003.

67. Billeter J.C., Rideout E.J., Dornan A.J. and Goodwin S.F., Control of male sexual behavior in Drosophila by the sex determination pathway. Curr. Biol. 16(17) 766-776, 2006.

68. Stockinger P., Kvitsiani D., Rotkopf S., Tirian L. and Dickson B.J., Neural circuitry that governs Drosophila male courtship behavior. Cell 121(5) 795-807, 2005. 
69. Manoli D.S., Foss M., Villella A., Taylor B.J., Hall J.C. and Baker B.S., Male-specific fruitless specifies the neural substrates of Drosophila courtship behaviour. Nature 436(7049) 395-400, 2005.

70. Vrontou E., Nilsen S.P., Demir E., Kravitz E.A. and Dickson B.J., fruitless regulates aggression and dominance in Drosophila. Nat. Neurosci. 9(12) 1469-1471, 2006.

71. Choi G.B., Dong H.W., Murphy A.J., Valenzuela D.M., Yancopoulos G.D., Swanson L.W. and Anderson D.J.,
Lhx6 delineates a pathway mediating innate reproductive behaviors from the amygdala to the hypothalamus. Neuron 46(4) 647-660, 2005.

72. Stowers L., Holy T.E., Meister M., Dulac C. and Koentges G., Loss of sex discrimination and male-male aggression in mice deficient for TRP2. Science 295(5559) 1493-1500, 2002 . 